\title{
Informed dispersal of the dandelion
}

\author{
Madeleine Seale ${ }^{1-3^{*}}$, Oleksandr Zhdanov ${ }^{4}$, Cathal Cummins ${ }^{1,2,5^{* *}}$, Erika Kroll ${ }^{1}$, Mike Blatt ${ }^{6}$, Hossein Zare-Behtash ${ }^{4}$, Angela \\ Busse $^{4}$, Enrico Mastropaolo ${ }^{3 \dagger}$, Ignazio Maria Viola ${ }^{5}$, and Naomi Nakayama ${ }^{1,2,7+* * *}$ \\ ${ }^{1}$ School of Biological Sciences, Institute of Molecular Plant Sciences, University of Edinburgh, EH9 3BF, UK \\ ${ }^{2}$ Centre for Synthetic and Systems Biology, University of Edinburgh, EH9 3BF, UK \\ ${ }^{3}$ School of Engineering, Institute for Integrated Micro and Nano Systems, University of Edinburgh, EH9 3FF, UK \\ ${ }^{4}$ James Watt School of Engineering, University of Glasgow, G12 8QQ, UK \\ ${ }^{5}$ School of Engineering, Institute for Energy Systems, University of Edinburgh, EH9 3DW, UK \\ ${ }^{6}$ Laboratory of Plant Physiology and Biophysics, Bower Building, University of Glasgow, G12 8QQ, UK \\ ${ }^{7}$ Centre for Science at Extreme Conditions, University of Edinburgh, EH9 3FD, UK \\ Present addresses \\ *Department of Plant Sciences. University of Oxford South Parks Road Oxford, OX1 3RB, UK \\ ${ }^{* * *}$ Maxwell Institute for Mathematical Sciences, Department of Mathematics and Institute for Infrastructure and Environment, Heriot-Watt University, Edinburgh, EH14 4AS \\ UK \\ **** Department of Bioengineering, Imperial College London, South Kensington, SW7 2AZ, UK \\ ${ }^{+}$Corresponding author.
}

\begin{abstract}
Animal migration is highly sensitised to environmental and biological cues, yet plant dispersal is considered largely passive. The common dandelion, Taraxacum officinale, is a classic example of a wind-dispersed plant and has an intricate haired pappus facilitating flight. This pappus facilitates enables the formation of a separated vortex ring (SVR) during flight (1); however, the pappus structure is not static but reversibly changes shape by closing in response to moisture. Here we characterise the biomechanical function of the pappus morphing regarding SVR dynamics and flight capacity. When the pappus closes, the falling velocity is greatly increased and the velocity deficit within the vortex decreased. To understand the implications of this structural-functional change, we used historic meteorological data to simulate dispersal distance. Dispersal distances were reduced with the pappus closed, and so was detachment. We propose that moisture-dependent pappus-morphing serves to retain seeds in favourable moist niches, providing a form of informed dispersal (2) that has not been characterised in plants on such a short-term, responsive time scale (3).
\end{abstract}

wind dispersal | biological fluid mechanics | abscission bias | dandelion | Asteraceae

Correspondence: n.nakayama@imperial.ac.uk

† Sadly deceased 15 July 2019 during preparation of this manuscript.

Results and Discussion. A long-distance shift in habitat is one of the riskiest decisions a living organism can make. From ballooning spiders dispersing from salt marshes according to local wind velocity (4) to kittiwakes abandoning breeding sites based on reproductive success of their neighbours (5), migration of many animals can be described as 'informed dispersal', in which organisms chose when and to where they travel depending on environmental cues and stressors (2). In contrast, movement of plants, which is mostly mediated by seed or fruit dispersal, is understood as a mostly passive process. This contradicts the wide range of environmental sensing and responses observed in plant development and physiology. Is plant dispersal really passive?

The dandelion represents diverse plant species that generate plumed types of wind-dispersed diaspores, thought to fly over remarkable distances (e.g. up to 100 miles (6)). The diaspore consists of an achene (fruit) attached to a pappus comprising around 100 filaments (1), which is found closed in wetting

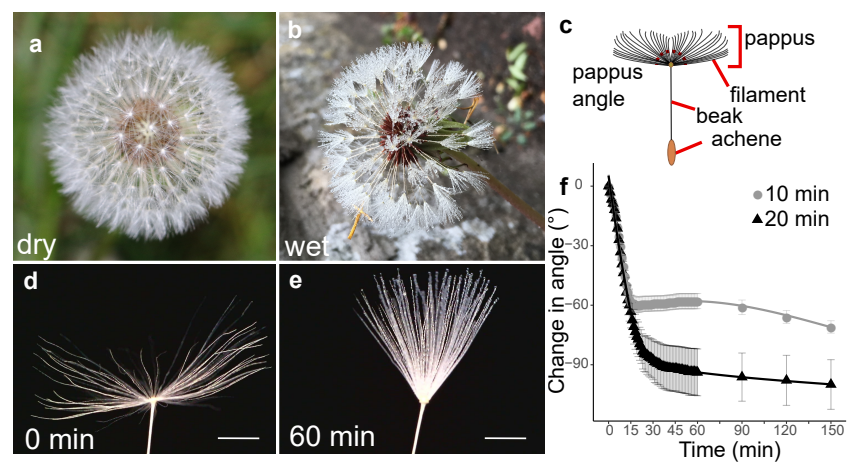

Fig. 1. Moisture induces reversible closure of the dandelion pappus. a image of a dry dandelion infructescence, $\mathbf{b}$ image of a wet dandelion infructescence, c schematic of a dandelion diaspore (seed) indicating features, $\mathbf{d}$ image of dry dandelion pappus, scale bar $=2 \mathrm{~mm}$. e image of dandelion pappus after 1 hour in moisture chamber with a 20 min humidifier treatment, scale bar $=2 \mathrm{~mm}$. $f$ time course of change in pappus angle between outermost filaments with 10 or $20 \mathrm{~min}$ humidifier treatment, $n=10$ and $n=12$ samples for 10 and 20 min treatments respectively, error bars are s.e.m.

conditions such as mist or rain $(7,8)$ (Fig 1a,b,c). In contrast, when dry, filaments are approximately horizontal at a $2 \mathrm{~mm}$ radius from the centre of the pappus, according to $\mu \mathrm{CT}$ scan analyses (Fig S1a).

To capture pappus closure dynamics, we imaged dandelion seeds in an enclosed, water-tight chamber with moisture added via an ultrasonic humidifier for 10 or 20 minutes (Fig $1 \mathrm{~d}, \mathrm{e}, \mathrm{f}$ ). For both treatments, the pappus angle (the angle between outermost filaments, see Fig 1c) rapidly decreased (4.0 $\pm 1.4^{\circ} \mathrm{min}^{-1}$ ) during moisture treatments. This continued for 5-15 minutes after moisture addition ceased with a final change in angle of $60^{\circ}$ to $90^{\circ}$ (Fig 1f). To test the effect of relative humidity $(\mathrm{RH})$ below $100 \%$, samples were placed in a chamber with stable RH. At RH of $71.8 \%$ and $87.0 \%$, modest angles changes of around $10^{\circ}$ and $24^{\circ}$ were observed, respectively (Fig S1b). Together, these results indicate that the dandelion pappus partially closes at high $\mathrm{RH}$ and rapidly closes when fully wet.

We predicted that pappus shape changes would modify seed flight and indeed found a strong effect on the falling velocity of the seed (Fig 2a). A decrease in pappus angle of around $100-150^{\circ}$, associated with wetting for one hour, resulted in 
bioRxiv preprint doi: https://doi.org/10.1101/542696; this version posted August 26,2020 . The copyright holder for this preprint (which was not certified by peer review) is the author/funder. All rights reserved. No reuse allowed without permission.

a

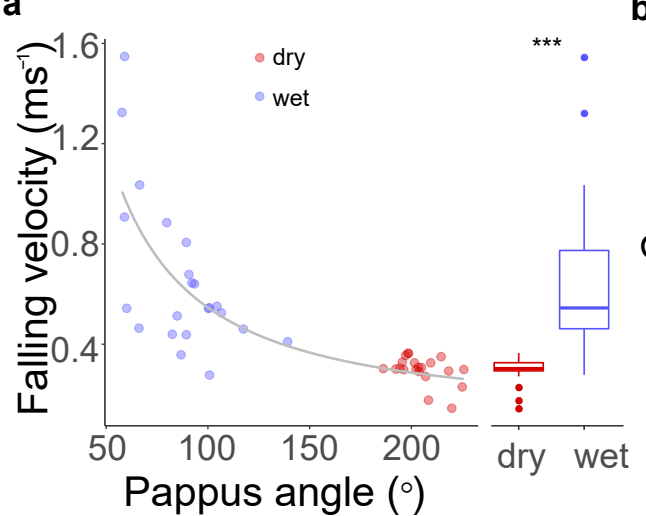

b

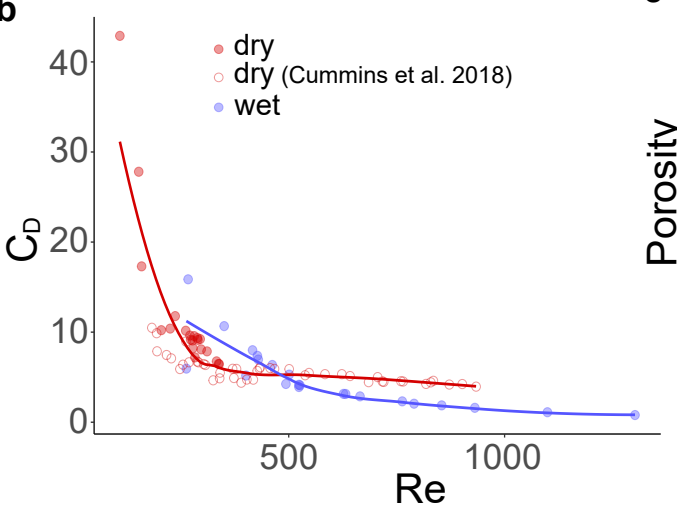

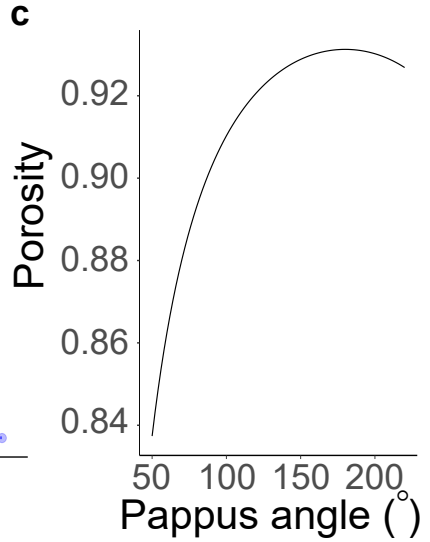

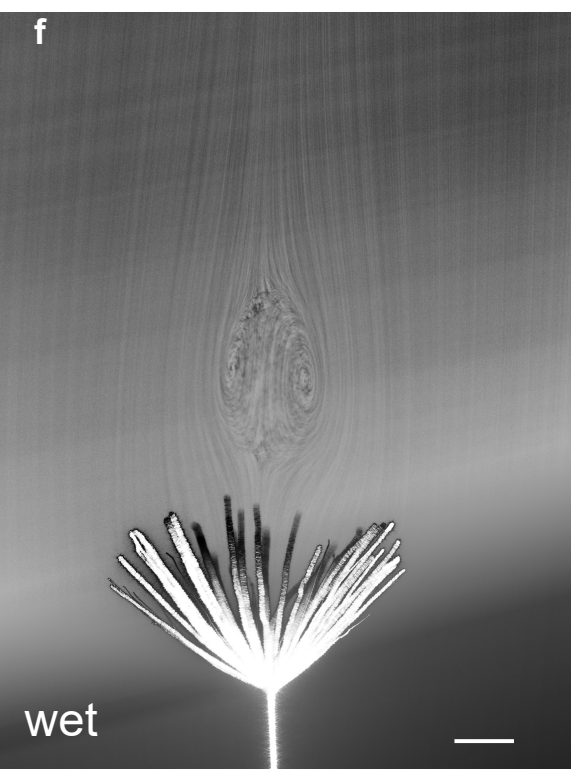

dry

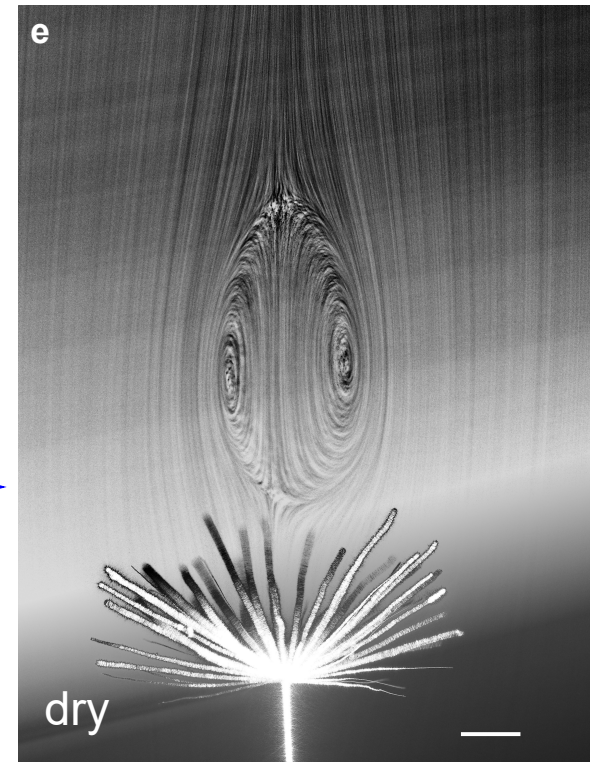

h

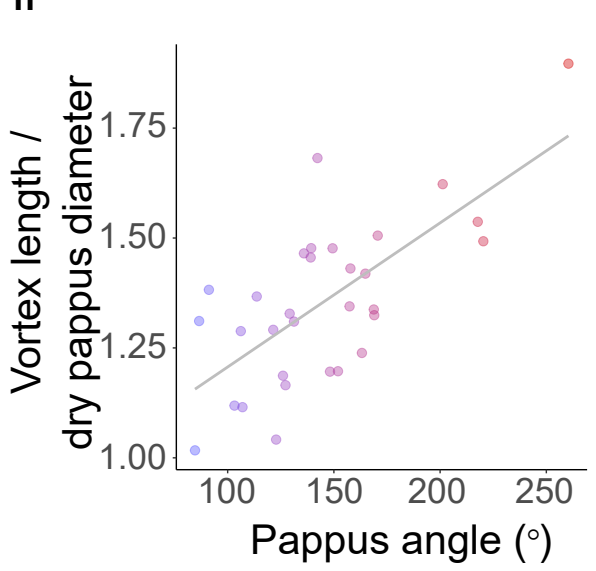

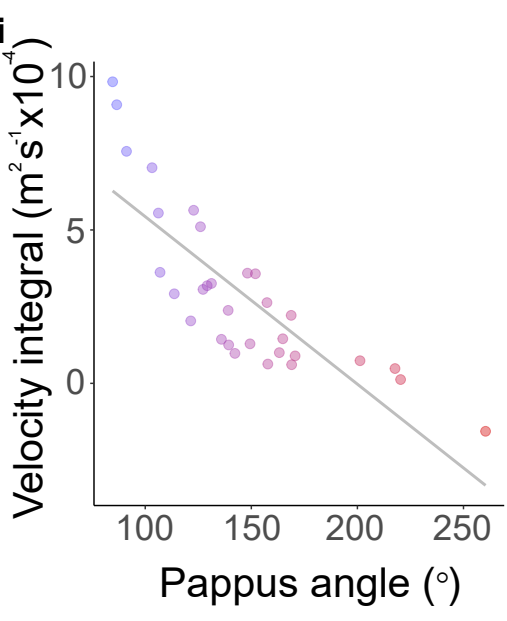

Fig. 2. Pappus morphing alters seed flight and fluid dynamics. a falling speed at varying pappus angles, before and after wetting for 1 hour in moisture chamber, asterisks indicate statistically significant difference at $p<0.0001, n=20$, b relationship between Reynolds number $(R e)$ and drag coefficient $\left(C_{D}\right)$ for dry and wet samples, open red circles indicate data from weighted/clipped dry seeds from ref (1), $n=10$ seeds, filled circles are same data as a, $n=20$ seeds, $\mathbf{c}$ estimated porosity at varying pappus angles, $\mathbf{d}$ schematic illustrating axes and direction of flow, e flow visualisation of dandelion fruit with open pappus, contrast adjusted to illustrate vortex more clearly, scale bar $=2 \mathrm{~mm}$, $\mathbf{f}$ flow visualisation of dandelion fruit with a partially closed pappus, contrast adjusted to illustrate vortex more clearly, scale bar $=2 \mathrm{~mm}$, $\mathbf{g}$ distance of the region of minimum $u_{z}$ from the pappus centre at varying pappus angle, position is nondimensionalised for each sample by the diameter of the pappus when dry, $n=12$ seeds, $\mathbf{h}$ longitudinal length of the SVR nondimensionalised by the diameter of the pappus when dry, at varying pappus angles, $n=12$ seeds, $\mathbf{i}$ integral of $u_{z}$ along a radial axis that intersects the $z$ axis at the point of maximal reverse flow, $n=12$ seeds. 
double to triple the falling velocity (Fig 2a). This is consistent with a change in projected pappus area that occurs when the pappus closes (Fig S2a). Additionally, a small increase in mass due to the added water resulted in a $0.7 \mu N$ increase in drag for the wetted samples.

To understand further how the pappus shape change affects the drag and falling velocity of the dandelion seed, we calculated the drag coefficient $\left(C_{D}\right)$ and Reynolds numbers $(R e)$ from our drop test data. The coefficient $C_{D}$ is a nondimensionalised indication of the drag on an object for a given projected area, while $R e$ is a nondimensionalised indication of the fluid regime, which depends only on fluid viscosity, speed and size of the object. For dry diaspores we examined $C_{D}$ and $R e$ alongside data from weighted/clipped seeds from ref (1) (Fig 2b). For dry diaspores, higher $R e$ is associated with rapidly decreasing $C_{D}$ before reaching a plateau. The wet diaspores with closed pappi, however, exhibit a different $C_{D^{-}}$ $R e$ relationship with substantially lower $C_{D}$ for a given $R e$ compared to dry (weighted) seeds with open pappi (Fig 2b). Closing the pappus therefore has two simultaneous effects: $R e$ is increased as falling velocity increases and the drag per unit of projected pappus area is decreased relative to a hypothetical open pappus of the same projected area. Therefore, pappus morphing is associated with shifting to a different region of the $C_{D^{-}}$Re parameter space.

A separated vortex ring (SVR) forms behind the pappus due to the high porosity of the pappus combined with precise spacing of the filaments, which limits air flow through the pappus and creates complex flow behaviour $(1,10)$. The nature of the SVR behind circular disks is strongly affected by the porosity of the disk and its projected area. Closing the pappus has several effects on the geometry: firstly, the projected area exposed to the flow reduces (Fig S2a); secondly, porosity of the pappus reduces as filaments move closer together (Fig 2c); and thirdly, the shape of the pappus becomes less disk-like and more cone-like. We have observed that the combination of these effects alters the fluid mechanics around the pappus by reducing vortex size, shifting the vortex away from the pappus, and increasing the velocity deficit in the wake (Fig 2g-i).

In order to understand how pappus morphing impacted on SVR formation, we examined dandelion pappi in dry and wet states fixed in a vertical wind tunnel (Fig 2d). Flow visualisation confirmed the presence of the SVR in both open and partially closed pappi (Fig 2e,f). We performed particle image velocimetry (PIV) (11) to spatially resolve the varying velocity of air in the region behind the pappus. Streamwise flow velocity was kept constant giving $R e=133-197$ across different pappus angles. Our PIV analysis was able to detect the expected features of the SVR (Fig 2g, S2b,c) (1).

The velocity deficit behind porous disks is associated with negative pressure (12). A larger vortex with a greater velocity deficit may therefore increase the magnitude of the negative pressure, enhancing drag on the pappus. In the region of space directly behind the centre of the pappus, the magnitude of minimum streamwise flow velocity (minimum $u_{z}$ ) showed no clear relationship with the pappus angle (Fig S2d).
However, the location of minimum $u_{z}$ correlated with pappus angle (Fig 2g, S2e). At more open pappus angles, the position of minimum $u_{z}$ moved further downstream of the pappus (Fig 2g, $p<0.001, R^{2}=0.34$ ).

We obtained $u_{z}$ velocity profiles in the $r$ direction of the pappus at the location where $u_{z}$ was minimal (e.g. Fig $\mathrm{S} 2 \mathrm{~b}$ ). The integral of a fixed central region of this velocity profile illustrates the magnitude of the velocity deficit in the wake of the pappus, with smaller values indicating a greater velocity deficit. This deficit is closely related to the drag force on the pappus. A significant negative relationship $\left(p<0.001, R^{2}=0.65\right)$ was observed between pappus angle and the velocity integral (Fig 2i) indicating that a greater volume of air is moving more slowly behind the pappus when it is more open. Furthermore, the size of the vortex significantly changes with altered pappus angle. The nominal length (see Methods) of the vortex was markedly reduced at smaller pappus angles (Fig $2 \mathrm{~h}, p<0.001, R^{2}=0.46$ ).

As drag and falling velocity are strongly affected by moisture-dependent pappus morphing, we expected pappus closure would influence dispersal distances. We modelled the effects of varying falling velocity on dispersal distances in various weather conditions using hourly meteorological data for 30 years of the summer/autumn period (the period of dandelion reproduction) at 5 locations in the UK (Edinburgh, Manchester, Nottingham, Norwich, and Exeter). Wind speeds at the height of a dandelion infructescence were inferred as described by (13), assuming a release height of $35 \mathrm{~cm}$ from the ground (9). For Edinburgh, the hourly wind speeds varied around a mean of $4.0 \mathrm{~m} \mathrm{~s}^{-1}$ (Fig 3a) (measured $10 \mathrm{~m}$ above ground, equivalent to $0.7 \mathrm{~m} \mathrm{~s}^{-1}$ at dandelion height). We inputted the hourly wind speed data into the well-described analytical WALD model that predicts seed dispersal distances (14), with varying falling velocities according to weather conditions (Fig 3d-f, S3e-p). At high RH ( $\mathrm{RH}=90 \%$ used as dry/wet cut off), when dandelion pappi are more likely to be closed, wind speeds are relatively low, yet still variable (Fig 3a, S3a-e). Plants that disperse with a morphing pappus (open in dry conditions, closed when wet) show reduced median dispersal compared to those that are always open regardless of the weather (median $=0.60$ vs 0.67 $\mathrm{m}$ ). Long distance dispersal (defined here as the furthest $1 \%$ of dispersal distances) is also slightly reduced (Fig 3e) (median $=3.78$ vs $3.90 \mathrm{~m}$ ). These patterns were similar in all geographical locations examined (Fig 3d,e, S3e-h,m-p).

From our earlier observations, we initially predicted that pappus closure would act to alter dispersal distances. However, our modelling showed only modest changes to both median dispersal distances and long distance dispersal (Fig 3d,e). Despite this, the suitability of locations for those seeds may not be equal. Considering only the dispersal of seeds in wet conditions, dispersal distances of seeds with a closed pappus are much more likely to remain close to the parent where it is wet, compared to those dispersing with an open pappus (Fig 3d-f).

Based on aerodynamic considerations, we also predicted pappus closure may affect detachment from the parent plant 

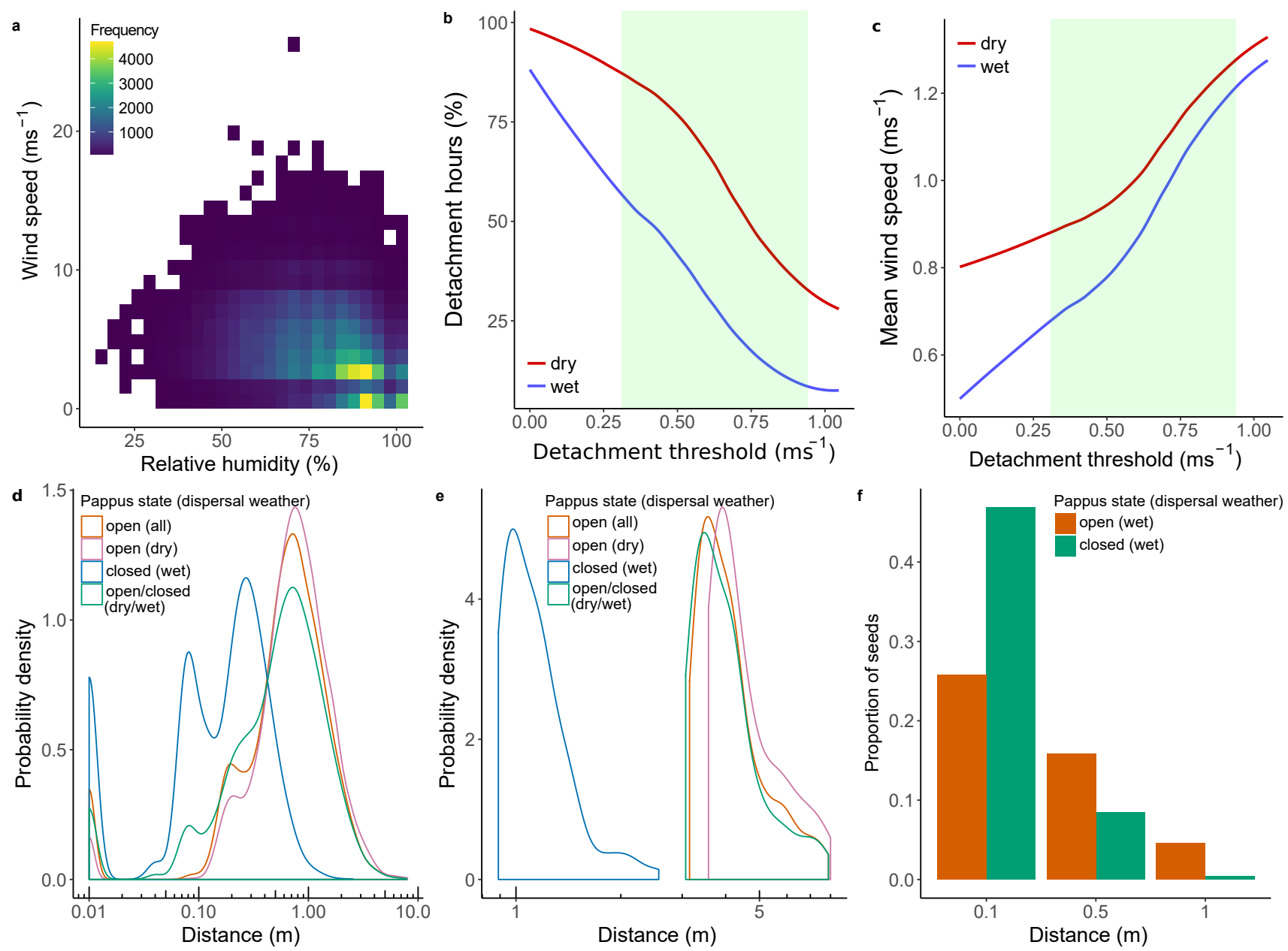

Fig. 3. Change in pappus angle modifies flight according to meteorological conditions. a relationship between relative humidity and wind speed in Edinburgh, UK, $\mathbf{b}$ fraction of time in which detachment could occur for dry conditions (red line, relative humidity less than $90 \%$ ) or wet conditions (blue line, relative humidity greater than $90 \%$ ) at varying detachment thresholds, green shaded area indicates the observed detachment range from ref (9) corrected to dandelion height, c mean wind speed at dandelion height above detachment threshold for dry conditions (red line) and wet conditions (blue line) at varying detachment threshold, $\mathbf{d}$ kernel density estimate of predicted dispersal distances for seeds with open or closed pappi and for different weather conditions, 'dry' indicates that only wind speeds observations for which humidity was less than $90 \%$ were used, 'wet' indicates the inverse, and 'dry/wet' corresponds to varying falling velocities depending on the humidity e kernel density estimate of predicted dispersal distances of furthest $1 \%$ of seeds in same conditions as $\mathbf{d}$, $\mathbf{f}$ proportion of seeds travelling short distances in wet conditions with the pappus open or pappus closed.

as the wind force required to detach seeds would increase. Correlations between detachment, higher wind speeds and low relative humidity have been observed for various winddispersed species during daily $(15)$ and seasonal $(16,17)$ humidity cycles, indicating dry seed release may be generally beneficial. Field observations previously showed that dandelion seed detachment correlated with hourly wind speeds at $20 \mathrm{~m}$ height of 2-6 $\mathrm{m} \mathrm{s}^{-1}$, which is equivalent to $0.31-0.94$ $\mathrm{m} \mathrm{s}^{-1}$ at dandelion height (9).

Using the meteorological data for all our tested locations, we analysed the fraction of time in which the wind speed is above the detachment threshold of $0.31-0.94 \mathrm{~m} \mathrm{~s}^{-1}$, and thus in which detachment could occur in either dry and or wet conditions. For dry conditions, a detachment threshold of $0.31 \mathrm{~m}$ $\mathrm{s}^{-1}$ at dandelion height results in $87 \%$ of dry hours in which hourly wind speeds are above threshold (Fig 3b), and a mean hourly wind speed for these hours of $0.9 \mathrm{~m} \mathrm{~s}^{-1}$ (Fig 3c). Assuming detachment occurs less easily in wet conditions, a threshold at the upper end of the observed range $\left(0.94 \mathrm{~m} \mathrm{~s}^{-1}\right)$, results in hourly wind speeds above threshold in just $9.7 \%$ of wet hours (Fig 3e) with a mean hourly wind speed of 1.2 $\mathrm{m} \mathrm{s}^{-1}$ (Fig 3c). This indicates that reduced seed detachment when wet may generally prevent dispersal initiation during times with low wind speeds.

Force thresholds on detachment are an important mechanism by which plants can bias timing of dispersal in favour of increased wind speeds $(9,15,18-22)$ and is thought to promote long distance dispersal $(19,21,22)$. Since our meteorological analysis indicated that the likelihood of seed detachment may be affected by humidity, we tested detachment experimentally in a wind tunnel. We placed the infructescence in a wind tunnel (Fig S4a) and assessed the number of seeds detaching at varying wind speeds (Fig 4). Hydrating samples for one hour significantly ( $p<0.001, \chi^{2}=341, d f=1$ ) reduced detachment for the range of wind speeds tested (Fig 4c, Fig S4b). In flow with $9.1 \%$ turbulent intensity, $24 \%$ of dry diaspores remained attached at the maximum wind speed $\left(9.8 \mathrm{~m} \mathrm{~s}^{-1}\right)$, compared to $50 \%$ of wet diaspores (Fig 4c). Re- 

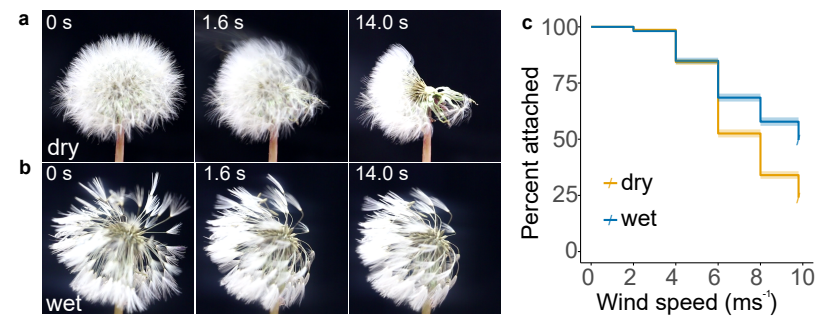

Fig. 4. Detachment of seeds from capitula. a snapshots of detachment at $8 \mathrm{~m} \mathrm{~s}^{-1}$ wind speed for a dry capitulum, $\mathbf{b}$ snapshots of detachment at $8 \mathrm{~m} \mathrm{~s}^{-1}$ wind speed for a wet capitulum, c survival plot of percentage of fruits attached to capitula at varying wind speeds for $9.1 \%$ turbulent intensity flow in dry and wet conditions, tested 10 14 days after opening of capitula, $n=20$ capitula per treatment corresponding to 3001 and 2654 seeds for dry and wet conditions respectively, shading around lines indicates s.e.m.

ducing the turbulent intensity of the flow also reduced seed detachment (Fig S4b,c). These data indicate that hydration and air flow impact on the initiation of dispersal, consistent with detachment data for thistles $(20,23,24)$.

Dispersing far from a parent is often considered favourable as resource competition is reduced, but this is not the only factor affecting niche suitability. Soil moisture levels are temporally and spatially variable (25), more than characteristics such as light and temperature (26), meaning that optimal germination sites and times may vary considerably with changing weather and microenvironments. Adult dandelion plants occupy a quite variable hydrological niche, indicating that they tolerate an environment with appreciable frequencies of both flooding and drought (27). Seedlings are more sensitive to hydrological fluctuations but germinate well in high moisture conditions (28).

Moisture-induced pappus morphing may thus be a form of informed dispersal, in which dispersal is directed according to environmental cues signalling more favourable conditions (2). In contrast to the popular perception that plant migration is a passive response to wind and other carriers, our extensive literature review revealed otherwise (3). Plants are not able to make conscious decisions influencing their behaviour, but can form mechanical or chemical structures that respond differentially to the environment. There are two types of informed dispersal in plants: developmental adaptation of heteromorphic seeds to environmental stresses (29), which acts over a longer time-scale responding to stable environmental conditions, and a few examples that are highly temporal and local as observed here. Such short-term regulation may be particularly beneficial in responding to unpredictable or fast changing conditions.

We have characterised the moisture-induced changes to structural functions of dandelion diaspores, a phenomenon that occurs in many (possibly most) pappus-bearing diaspores $(7,8)$. We have tested the ecological implications of this biomechanical response to moisture concluding that pappus closure acts to tune dispersal, preventing long distance dispersal when local conditions are favourable. As many species modify their seed or fruit structure in response to water, this may be a general mechanism to ensure dispersal at appropriate times. Considering how prevalent environmentally in- duced plasticity is in plant structure and its development, understanding of structural functions within environmental contexts is critical to predict the ecological consequences of climate change (30). Insights into ecological and environmental drivers in turn enable us to comprehend selective pressure that has acted and will act to evolve plant forms and their functions.

\section{ACKNOWLEDGEMENTS}

We thank Alex Twyford, Jonathan Silvertown and Tomasz Zielinksi for critical reading of the manuscript. We also thank Kazan Federal University Library for providing access to the 1894 manuscript by Taliev (8).

\section{COMPETING FINANCIAL INTERESTS}

The authors declare no competing financial interests.

\section{Bibliography}

1. Cathal Cummins, Madeleine Seale, Alice Macente, Daniele Certini, Enrico Mastropaolo, Ignazio Maria Viola, and Naomi Nakayama. A separated vortex ring underlies the flight of the dandelion. Nature, 562:414-418, 2018. doi: doi.org/10.1038/s41586-018-0604-2.

2. Jean Clobert, Jean-François Le Galliard, Julien Cote, Sandrine Meylan, and Manuel Massot. Informed dispersal, heterogeneity in animal dispersal syndromes and the dynamics of spatially structured populations. Ecology letters, 12(3):197-209, 2009. ISSN 1461-0248. doi: 10.1111/j.1461-0248.2008.01267.x.

3. Madeleine Seale and Naomi Nakayama. From passive to informed: mechanical mechanisms of seed dispersal. New Phytologist, 225(2):653-658, Jan 2020. ISSN 0028-646X. doi: 10.1111/nph.16110.

4. Dries Bonte, Beatrijs Bossuyt, and Luc Lens. Aerial dispersal plasticity under different wind velocities in a salt marsh wolf spider. Behavioral Ecology, 18(2):438-443, Dec 2006. ISSN 1045-2249. doi: 10.1093/beheco/arl103.

5. Thierry Boulinier, Karen D McCoy, Nigel G Yoccoz, Julien Gasparini, and Torkild Tveraa. Public information affects breeding dispersal in a colonial bird: kittiwakes cue on neighbours. Biology Letters, 4(5):538-540, Oct 2008. doi: 10.1098/rsbl.2008.0291.

6. James Small. The origin and development of the compositae. New Phytologist, 17(8/9): 200-230, 1918. ISSN 0028646X, 14698137.

7. W. Rothert. On the hygroscopic tissue of the compositae pappus [in German]. Botanisches Zentralblatt, 63:320-324, 1895.

8. V Taliev. On the hygroscopic tissue of the compositae pappus. [in Russian]. Proceedings of the Society of Naturalists at the Imperial University of Kazan, 27(3):1-38, 1894

9. Jelle Treep, Monique de Jager, Leandra S Kuiper, Gabriel G Katul, and Merel B Soons. Costs and benefits of non-random seed release for long-distance dispersal in winddispersed plant species. Oikos, 127:1330-1343, 2018. doi: 10.1111/oik.04430.

10. P G Ledda, L Siconolfi, F Viola, S Camarri, and F Gallaire. Flow dynamics of a dandelion pappus: A linear stability approach. Physical Review Fluids, 4(7):71901, Jul 2019. doi: 10.1103/PhysRevFluids.4.071901.

11. Markus Raffel, Christian E. Willert, Fulvio Scarano, Christian J. Kähler, Steve T. Wereley, and Jürgen Kompenhans. Particle Image Velocimetry A Practical Guide. Springer, 3 edition, 2018. doi: 10.1007/978-3-319-68852-7.

12. Cathal Cummins, Ignazio Maria Viola, Enrico Mastropaolo, and Naomi Nakayama. The effect of permeability on the flow past permeable disks at low reynolds numbers. Physics of Fluids, 29(9):97103, Sep 2017. ISSN 1070-6631. doi: 10.1063/1.5001342.

13. Olav Skarpaas and Katriona Shea. Dispersal patterns, dispersal mechanisms, and invasion wave speeds for invasive thistles. The American Naturalist, 170(3):421-430, 2007. doi: $10.1086 / 519854$.

14. G G Katul, A Porporato, R Nathan, M Siqueira, M B Soons, D Poggi, H S Horn, and S A Levin. Mechanistic analytical models for long-distance seed dispersal by wind. The American Naturalist, 166(3):368-381, 2005.

15. D F Greene, M Quesada, and C Calogeropoulos. Dispersal of seeds by the tropical sea breeze. Ecology, 89(1):118-125, 2008

16. Felix Heydel and Oliver Tackenberg. How are the phenologies of ripening and seed release affected by species' ecology and evolution? Oikos, 126:738-747, 2017. doi: 10.1111/oik. 03442

17. S Joseph Wright, Ana Trakhtenbrot, Gil Bohrer, Matteo Detto, Gabriel G Katul, Nir Horvitz, Helene C Muller-landau, Frank A Jones, and Ran Nathan. Understanding strategies for seed dispersal by wind under contrasting atmospheric conditions. Proceedings of the $\mathrm{Na}$ tional Academy of Sciences of the United States of America, 105(49):19084-19089, 2008. doi: 10.1073/pnas.0802697105.

18. David $\mathrm{F}$ Greene. The role of abscission in long-distance seed dispersal by the wind. Ecology, 86(11):3105-3110, 2005.

19. Peter Schippers and Eelke Jongejans. Release thresholds strongly determine the range of seed dispersal by wind. Ecological Modelling, 185:93-103, 2005. doi: 10.1016/j.ecolmodel. 2004.11.018.

20. Olav Skarpaas, Richard Auhl, and Katriona Shea. Environmental variability and the initiation of dispersal: turbulence strongly increases seed release. Proceedings of the Royal Society B, 273:751-756, 2006. doi: 10.1098/rspb.2005.3366.

21. Merel B Soons and James M Bullock. Non-random seed abscission, long-distance wind dispersal and plant migration rates. Journal of Ecology, 96:581-590, 2008. doi: 10.1111/j. 1365-2745.2007.0.

22. Sally E Thompson and Gabriel G. Katul. Implications of nonrandom seed abscission and global stilling for migration of wind-dispersed plant species. Global Change Biology, 19: 1720-1735, 2013. doi: 10.1111/gcb.12173. 
bioRxiv preprint doi: https://doi.org/10.1101/542696; this version posted August 26,2020 . The copyright holder for this preprint (which was not certified by peer review) is the author/funder. All rights reserved. No reuse allowed without permission.

23. Eelke Jongejans, Nicholas M Pedatella, Katriona Shea, Olav Skarpaas, and Richard Auhl. Seed release by invasive thistles: the impact of plant and environmental factors. Proceedings of the Royal Society B, 274:2457-2464, 2007. doi: 10.1098/rspb.2007.0190.

24. Katherine M Marchetto, Eelke Jongejans, Katriona Shea, and Richard Auhl. Water loss from flower heads predicts seed release in two invasive thistles. Plant Ecology \& Diversity, 5(1):57-65, 2012. doi: 10.1080/17550874.2012.667841.

25. Konstantin Y Vinnikov, Alan Robock, Nina A Speranskaya, and C Adam Schlosser. Scales of temporal and spatial variability of midlatitude soil moisture. Journal of Geophysical Research: Atmospheres, 101(D3):7163-7174, Mar 1996. ISSN 0148-0227. doi: 10.1029/95JD02753.

26. Jonathan Silvertown, Yoseph Araya, and David Gowing. Hydrological niches in terrestrial plant communities: a review. Journal of Ecology, 103(1):93-108, Oct 2014. ISSN 00220477. doi: $10.1111 / 1365-2745.12332$.

27. Jonathan Silvertown, Mike E Dodd, David J G Gowing, and J Owen Mountford. Hydrologically defined niches reveal a basis for species richness in plant communities. Nature, 400 : 61, Jul 1999.

28. Nathan S Boyd and Rene C Van Acker. The effects of depth and fluctuating soil moisture on the emergence of eight annual and six perennial plant species. Weed Science, 51(5): 725-730, 2003. ISSN 00431745, 15502759.

29. Carlos Martorell and Marcela Martínez-López. Informed dispersal in plants: Heterosperma pinnatum (asteraceae) adjusts its dispersal mode to escape from competition and water stress. Oikos, 123(2):225-231, Feb 2014. ISSN 0030-1299. doi: 10.1111/j.1600-0706. 2013.00715.x.

30. Mark Denny and Brian Helmuth. Confronting the physiological bottleneck: a challenge from ecomechanics. Integrative and Comparative Biology, 49(3):197-201, 2009. doi: 10.1093/ icb/icp070. 


\section{Methods.}

Plant growth and samples. Taraxacum officinale samples were collected and grown as described previously (1). All samples were the progeny of plants grown in the greenhouse for 2 generations originating from the same individual. As this subspecies reproduces apomictically, all seeds used were considered to be genetically identical. For detachment assays, dandelions were grown in the greenhouse and stems with capitula harvested once the inflorescence had closed but before the infructescence had opened. $15 \mathrm{ml}$ tubes were filled with water and covered by a piece of parafilm with a small hole in and the stems of samples placed through the hole. These samples were then placed in a greenhouse (ambient conditions except for day length, which is artificially extended with electric lighting to ensure at least 16 hours of daylight) to allow the infructescence (capitulum) to open.

Pappus angle measurements. Source data and analysis of 3D micro-tomography CT scans were identical to ref (1). Angles of each filament from the horizontal axis was calculated at a $2 \mathrm{~mm}$ radius from the centre of the pappus, with the beak aligned to the vertical axis. For all other pappus angle measurements, the angle was measured from 2D microscope or camera images. The pappus was aligned perpendicular to the objective/lens and pappus angle measured as the angle between the outermost filaments.

Moisture chamber imaging. The moisture chamber consisted of a $70 \mathrm{~L}$ airtight plastic box (Solent Plastics, UK). A hole was made in the box to pass cables through and the space around sealed with silicone sealant. Two USB microscopes (Maozua, USB001) were positioned horizontally to image dandelion seed samples, which were fixed in place by embedding in plasticine or individual pieces of foil shaped around the achene. An ultrasonic humidifier (Bottle Caps) was filled with distilled water and placed next to the dandelion samples. The ultrasonic humidifier releases small droplets of liquid water into the chamber meaning that relative humidity (RH) reaches $100 \%$ within the first few minutes. Samples were imaged each minute for the duration of the experiment. For relative humidities below $100 \%$, dishes of saturated salt solutions were placed into the chamber to maintain defined, stable humidity levels (2). Relative humidity was monitored with a datalogger (Lascar, Easylog EL-GFX-2). Samples were imaged at 0 hours and 10 hours, when RH had long reached equilibrium.

Falling velocity assays. Drop tests were carried out using a modified version to the protocol in ref (1). Samples were tested in two batches $(n=10$ and $n=11)$. First, all seeds were weighed together to obtain the mean weight per sample. Each sample was dropped 3 times and video recorded with a DSLR camera (Canon). Samples were then imaged in the moisture chamber while still dry. The humidifier was switched on for 1 hour and samples re-imaged. Seeds were removed from the chamber one by one and dropped 3 times before placing back inside the humid chamber to prevent further drying. After all samples were dropped, all were weighed together again. The mean increase in weight after wetting was $70 \mu \mathrm{g}$, which corresponds to approximately $10 \%$ greater weight when wet compared to dry. Falling velocity was determined from the video frames using a particle detection script (1).

Calculated variables. The projected area was calculated according to the pappus angle, assuming 100 straight, nonoverlapping filaments of length $7.41 \mathrm{~mm}$ and diameter 16 $\mu \mathrm{m}$. Porosity was calculated as the ratio of empty space between filaments (i.e. 1 - projected area) to the total area of the circle that would enclose the projected area.

The Reynolds number is defined as $R e=\frac{u D}{\nu}$, where $u$ is the flow velocity (the falling velocity, i.e. the undisturbed velocity of the fluid with respect to the pappus), $D$ is the characteristic length scale (in our case, this is the diameter of the pappus assuming filament length of $7.41 \mathrm{~mm}$ and varies according to pappus angle) and $\nu$ is the kinematic viscosity of the fluid at $20^{\circ} \mathrm{C}$.

The drag coefficient was calculated as follows:

$$
C_{D}=\frac{m g}{\frac{1}{2} \rho u^{2} A}
$$

where $\rho=1.0241 \mathrm{~kg} \mathrm{~m}^{-3}$ is the density of air, $A$ is the projected area of the disk, taking into account porosity, and $g=9.81 \mathrm{~m} \mathrm{~s}^{-1}$ is gravitational acceleration. Additionally, $m$ is the mass of the seed, for which an average measured value of $0.614 \mathrm{mg}$ was used for all dry samples and $0.684 \mathrm{mg}$ for all wet samples.

\section{Particle image velocimetry (PIV) and flow visualisa-} tion. Samples were fixed in place inside a vertical wind tunnel for flow visualisation and PIV (1). Long exposure images were obtained using a Canon DSLR camera (EOS 70D). The raw experimental images were processed to distinguish the region of interest (i.e. the SVR) from the reflective pappus. To do this, we applied a digital graduated neutral density filter using the "Curves" contrast adjust tool and the "Blend" tool within the open source software GNU Image Manipulation Program (GIMP version 2.8.22).

For PIV, videos were obtained with a high-speed camera (Fastcam Photron SA1.1) shooting at 125 frames per second fitted with a macro lens (Tamron). Air flow velocity was $0.207 \mathrm{~m} \mathrm{~s}^{-1}$ throughout. 100 frames of each video were analysed in PIVlab (Matlab) (3) using single pass direct cross correlation with a window size of 128 pixels (corresponding to $3.88 \mathrm{~mm}$ ) and a window overlap of 64 pixels. Data were filtered by excluding vectors of more than 2 standard deviations and a normalised median filter (4) with a minimum normalisation level $(\epsilon)$ of 0.1 pixels and a detection threshold of 3. Vectors were interpolated where missing and the mean vector field of all 50 pairs of images was calculated.

Noisy rows of data from the images were excluded from further analysis by removing the first row of the image (row size defined by the window size), which was always noisy, and identifying other noisy rows by fitting an autoregressive integrated moving average model ( $\mathrm{R}$ package 'tsoutliers') to 
the profiles of streamwise velocity (uz) across the $r$ direction of the image. The velocity profiles with outliers were considered noisy. A $z$ portion of the image with no more than one consecutive noisy row was considered to form the vortex region and was used for further analysis. Analysis was also limited in the $r$ direction by excluding regions of data that fell outside of the limits of the dandelion pappus width. The co-ordinates of the central point of the pappus and the pappus angle were measured from images. The pappus limits were calculated assuming the pappus formed a triangular shape with filaments that were $7.41 \mathrm{~mm}$ long (1).

The point $P$ of minimal flow was determined by finding where $u_{z}$ along the $z$-axis was minimal at the $r$ location of the centre of the pappus. Let $z_{p}$ be the $z$-coordinate of $P, D$ the diameter of the pappus and $\tau$ the pappus angle. We found that

$$
\frac{z_{p}}{D}=0.24+0.0039 \tau
$$

$\left(p<0.001, R^{2}=0.34\right)$.

Using this relationship (Fig 2f), we identified a nominal zcoordinate of minimal $u_{z}$ at which we measured the wake deficit. At this co-ordinate, $u_{z}$ profiles across the $r$ direction of the image were plotted (e.g. Fig S2b) and the following integral calculated (Fig 2h)

$$
\int_{0}^{1} u_{z} r d r
$$

to establish the extent of the velocity deficit in the wake of the pappus. Integrals were calculated for a fixed $10 \mathrm{~mm}$ window around the centre of each sample. This distance was chosen as it corresponds to the smallest observed pappus diameter in the dataset. The flow structure is not well resolved beyond the radial limit of the vortex (the frame rate is too low to capture the fast flow speed of the external regions) so this limited the $r$ range for the analysis.

Similarly, it was not possible to identify the downstream stagnation point where the velocity vanished and thus the nominal vortex length was determined by obtaining the point beyond the centre of the pappus at which $u_{z}=0.02 \mathrm{~m} \mathrm{~s}^{-1}$. This does not give the actual length of the vortex but gives an indicative value to compare between samples and treatments.

Meteorological data analysis. Hourly meteorological data was obtained from the Integrated Surface Database from the National Centers for Environmental Information (USA). Data for a 30 year period $(1987$ - 2017) were acquired for each location (Edinburgh $(55.950,-3.373)$, Norwich (52.676, 1.283), Nottingham (52.831, -1.328), Manchester (53.354, 2.275), Exeter $(50.734,-3.414))$ and filtered according the following criteria: entries were excluded if hourly wind speed, dewpoint or temperature were missing; data were only used from the beginning of April to the end of October in each year; occasional data measured between hours were excluded. Relative humidity was calculated from the dewpoint and temperature.
The release height of dandelion seeds was assumed to be 0.35 $\mathrm{m}$ according to ref (5). Wind speeds at this height were calculated by integrating the wind speed over a logarithmic profile following ref (6) and ref (7).

Dispersal modelling. The WALD model of wind dispersal was used to predict dandelion seed dispersal distances at varying terminal velocities (8). This is an analytical mechanistic model based on an inverse Gaussian distribution that uses hourly wind speeds and features of vegetation structure to predict dispersal kernels. Vegetation height around dandelions was taken to be $0.17 \mathrm{~m}$, a value obtained from LEDA, a database of Northwest European flora life history traits (9). We assumed surface roughness, friction velocity and turbulent flow to be similar to dispersal of thistle species (short herbaceous vegetation), so used the same values and calculation methods for these parameters as (6).

As dandelion pappi can partially close at RH $>70 \%$ (Fig S1b) and field-measured $\mathrm{RH}>90 \%$ is a good proxy for wetness on plant surfaces (10), we used $\mathrm{RH}=90 \%$ as a cut-off point between dry and wet conditions.

Seed falling velocities for dry and wet conditions were 0.3 and $0.7 \mathrm{~m} \mathrm{~s}^{-1}$ respectively, corresponding to the measured values from our drop tests.

Dispersal kernels were obtained for each location by selecting a time point at random, calculating the wind speed at dandelion height (as above) and selecting a dispersal distance according to the probability distribution given by the WALD function. This was repeated for 10,000 simulations for each model run giving an overall dispersal kernel for the weather conditions at that location.

Detachment assays. A modified version of the method in ref (11) was used to assess detachment of seeds from the capitulum. Tests were conducted in a horizontal, open-circuit wind tunnel. The test section was $1 \mathrm{~m}$ long with a rectangular cross-section of $450 \mathrm{~mm}$ x $400 \mathrm{~mm}$ (Fig S4a). Either 3 days (Fig S4b,c) or 10-14 days after opening (Fig 4c), samples were placed in the middle of the test section and were subjected to a flow speed of $2 \mathrm{~m} \mathrm{~s}^{-1}$ for 30 seconds. Samples were held in place by passing an aluminium rod (rod diameter $=1.5 \mathrm{~mm}$ or $3 \mathrm{~mm}$ depending on stem diameter) through the hollow stem attached to the capitulum or for older samples, in which the stem had degraded, were clamped to the rod. Detached seeds were collected in a mesh at the end of the test section (Fig S4a) and counted. This was repeated for 4, 6, 8 and $10 \mathrm{~m} \mathrm{~s}^{-1}$. After the maximum wind speed, the number of seeds remaining on the capitulum was also counted. For more turbulent conditions, a grid consisting of 11 equally spaced vertical rods (diameter $=10 \mathrm{~mm}$ ) was created and placed inside the test section $(200 \mathrm{~mm}$ downstream of the inlet). This increased turbulence intensity to $9.1 \%$, but the maximum wind speed was reduced slightly to $9.8 \mathrm{~m} \mathrm{~s}^{-1}$ and this was used instead of $10 \mathrm{~m} \mathrm{~s}^{-1}$. The turbulent intensities used here are comparable to that in some natural conditions (12), though turbulence in the atmospheric boundary layer exhibits enormous variability, with values as high as $400 \%$ reported (13). 
bioRxiv preprint doi: https://doi.org/10.1101/542696; this version posted August 26, 2020. The copyright holder for this preprint (which was not certified by peer review) is the author/funder. All rights reserved. No reuse allowed without permission.

Wet capitula were treated by placing in the moisture chamber with the humidifier switched on for one1 hour before placing in the wind tunnel.

Data analysis and statistics. All measurements were carried out on separate individual seeds from different infructescences except detachment assays, in which multiple seeds from each infructescence were counted.

Detachment assays were analysed by fitting Kaplan-Meier survival models to the data, with log-rank tests used to compare treatments ( $\mathrm{R}$ package 'survival'). For falling velocity tests, the following model was fitted to the data where: falling velocity $\sim \cos ^{-2 / 3} \tau$ similarly to ref (14) and dry and wet groups were compared using Student's 2-sample ttest. For PIV data, linear regression models were fit to the data. For the plot of the relationship between $C_{D}$ and $R e$, loess regression with a span of 0.8 was used to illustrate the trends in the data.

Data were inspected for normality where appropriate.

For boxplots, the centre line is the median, hinges indicate first and third quartiles, and whiskers extend to largest value no further than 1.5 times the interquartile range.

\section{Bibliography}

1. Cathal Cummins, Madeleine Seale, Alice Macente, Daniele Certini, Enrico Mastropaolo, Ignazio Maria Viola, and Naomi Nakayama. A separated vortex ring underlies the flight of the dandelion. Nature, 562:414-418, 2018. doi: doi.org/10.1038/s41586-018-0604-2.

2. A. Wexler and S. Hasegawa. Relative humidity-temperature relationships of some saturated salt solutions in the temperature range $0^{\circ}$ to $50^{\circ} \mathrm{C}$. Journal of Research of the National Bureau of Standards, 53(1):19-26, 1954. ISSN 0091-0635. doi: 10.6028/jres.053.003.

3. William Thielicke and Eize J. Stamhuis. Pivlab - towards user-friendly, affordable and accurate digital particle image velocimetry in matlab. Journal of Open Research Software, 2(1): e30, 2014. doi: doi.org/10.5334/jors.bl.

4. Jerry Westerweel and Fulvio Scarano. Universal outlier detection for piv data. Experiments in Fluids, 39(6):1096-1100, 2005. ISSN 1432-1114. doi: 10.1007/s00348-005-0016-6.

5. Jelle Treep, Monique de Jager, Leandra S Kuiper, Gabriel G Katul, and Merel B Soons. Costs and benefits of non-random seed release for long-distance dispersal in winddispersed plant species. Oikos, 127:1330-1343, 2018. doi: 10.1111/oik.04430.

6. Olav Skarpaas and Katriona Shea. Dispersal patterns, dispersal mechanisms, and invasion wave speeds for invasive thistles. The American Naturalist, 170(3):421-430, 2007. doi: $10.1086 / 519854$

7. James M Bullock, Steven M White, Christel Prudhomme, Christine Tansey, Ramón Perea, and Danny A P Hooftman. Modelling spread of british wind-dispersed plants under future wind speeds in a changing climate. Journal of Ecology, 100:104-115, 2012. doi: 10.1111/j. 1365-2745.2011.01910.x.

8. G G Katul, A Porporato, R Nathan, M Siqueira, M B Soons, D Poggi, H S Horn, and S A Levin. Mechanistic analytical models for long-distance seed dispersal by wind. The American Naturalist, 166(3):368-381, 2005.

9. M Kleyer, R M Bekker, I C Knevel, J P Bakker, K Thompson, M Sonnenschein, Poschlod P, J M van Groenendael, L Klimeš, J Klimešova, and et al. The leda traitbase: a database of life-history traits of the northwest european flora. Journal of Ecology, 96:1266-1274, 2008. doi: 10.1111/j.1365-2745.2008.01430.x.

10. Paulo C Sentelhas, Anna Dalla Marta, Simone Orlandini, Eduardo A Santos, Terry J Gillespie, and Mark L Gleason. Suitability of relative humidity as an estimator of leaf wetness duration. Agricultural and Forest Meteorology, 148(3):392-400, 2008. ISSN 0168-1923. doi: https://doi.org/10.1016/j.agrformet.2007.09.011.

11. Eelke Jongejans, Nicholas M Pedatella, Katriona Shea, Olav Skarpaas, and Richard Auhl. Seed release by invasive thistles: the impact of plant and environmental factors. Proceedings of the Royal Society B, 274:2457-2464, 2007. doi: 10.1098/rspb.2007.0190.

12. Farzin Ghanadi, Matthew Emes, Jeremy Yu, Maziar Arjomandi, and Richard Kelso. Investigation of the atmospheric boundary layer characteristics on gust factor for the calculation of wind load. AIP Conference Proceedings, 1850(1):130002, Jun 2017. ISSN 0094-243X. doi: $10.1063 / 1.4984496$

13. J. D. Wilson, D. P. Ward, G. W. Thurtell, and G. E. Kidd. Statistics of atmospheric turbulence within and above a corn canopy. Boundary-Layer Meteorology, 24:495-519, 1982. ISSN 00068314. doi: 10.1007/BF00120736.

14. Qing'an Meng, Qianbin Wang, Kaibin Zhao, Pengwei Wang, Peiqing Liu, Huan Liu, and Lei Jiang. Hydroactuated configuration alteration of fibrous dandelion pappi: Toward selfcontrollable transport behavior. Advanced Functional Materials, 26(41):7378-7385, Aug 2016. ISSN 1616-301X. doi: 10.1002/adfm.201602596. 


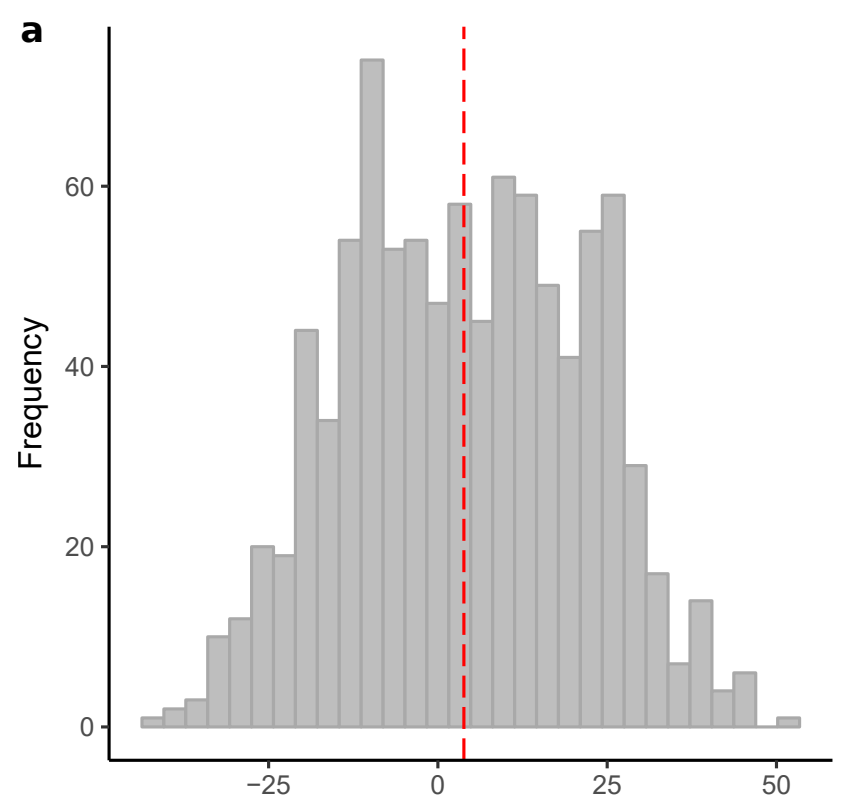

Filament angle from horizontal $\left({ }^{\circ}\right)$

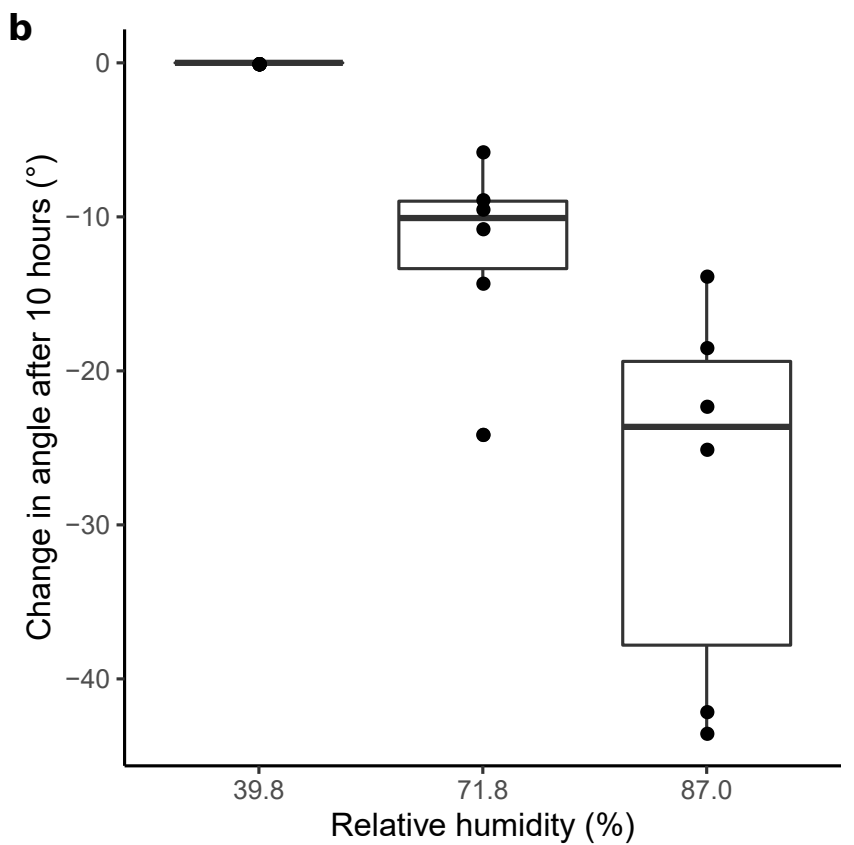

Fig. S1. Supplementary Figure 1. a the angle of individual filaments of dandelion pappi. Angle is calculated relative to horizontal in which the dandelion seed beak (see Fig 1c) forms the vertical axis, $n=10$ pappi, $n=932$ filaments. Red dashed line indicates the median value. $\mathbf{b}$ high relative humidity partially closes the dandelion pappus. Change in pappus angle at stable relative humidity (after 10 hours in chamber with saturated salt solutions), $n=7$ for $39.8 \%$ relative humidity and $n=6$ for $71.8 \%$ and $87.0 \%$ treatments. 
a

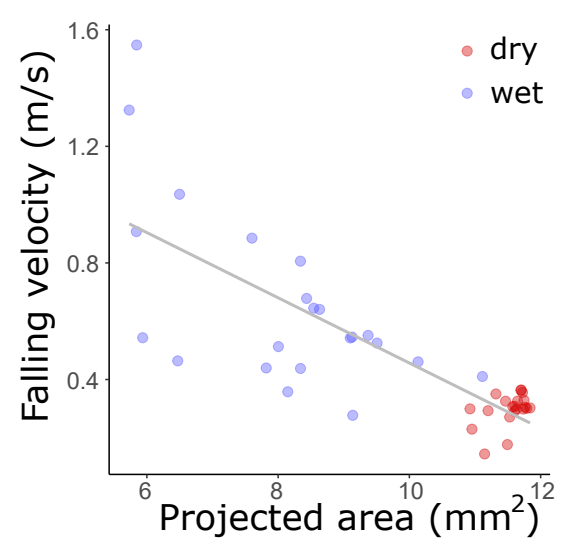

d

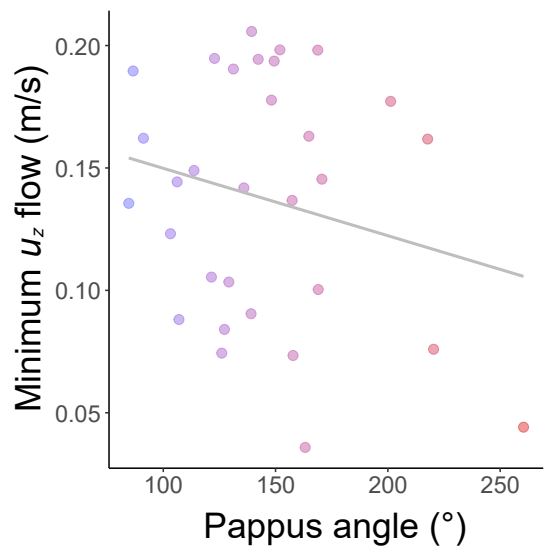

b

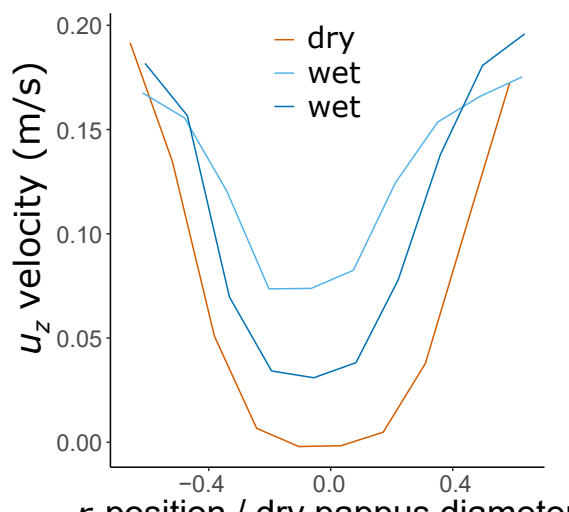

$r$ position / dry pappus diameter
C

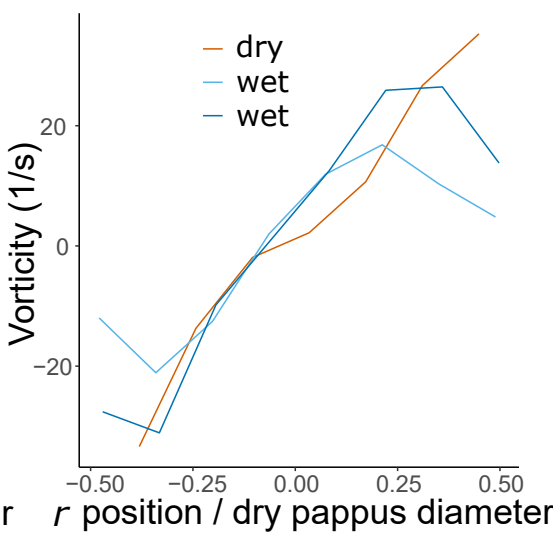

$\mathbf{e}$

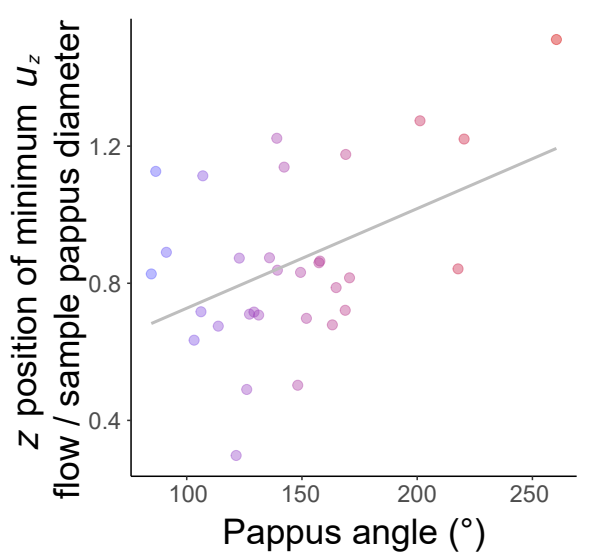

Fig. S2. Supplementary Figure 2. a falling velocity reduces as projected area reduces. Projected area calculated using measured pappus angles and assuming filament lengths of $7.41 \mathrm{~mm}$ and diameters of $16 \mu \mathrm{m}$ according to ref (1), $\mathbf{b}$ flow velocity along the $r$ axis of vortex images at calculated position of minimal $u_{z}$ flow. The $r$ position is non-dimensionalised by dividing by the dry pappus diameter for each sample, with 0 indicating the centre of the pappus. Plot for a single sample at 3 different pappus angles, representative of $n=10$ samples. c vorticity along the $r$ axis of vortex images at calculated position of minimal $u_{z}$ flow. The $r$ position is non-dimensionalised by dividing by the dry pappus diameter for each sample, with 0 indicating the centre of the pappus. Plot for a single sample at 3 different pappus angles, representative of $n=10$ samples. d the minimum $u_{z}$ flow velocity in the separated vortex ring at varying pappus angles. e the distance downstream of maximum reverse flow in the separated vortex ring at varying pappus angles. The $z$ position is relative to the sample pappus diameter (i.e. the diameter of the sample at the given pappus angle, rather than in a dry state). 

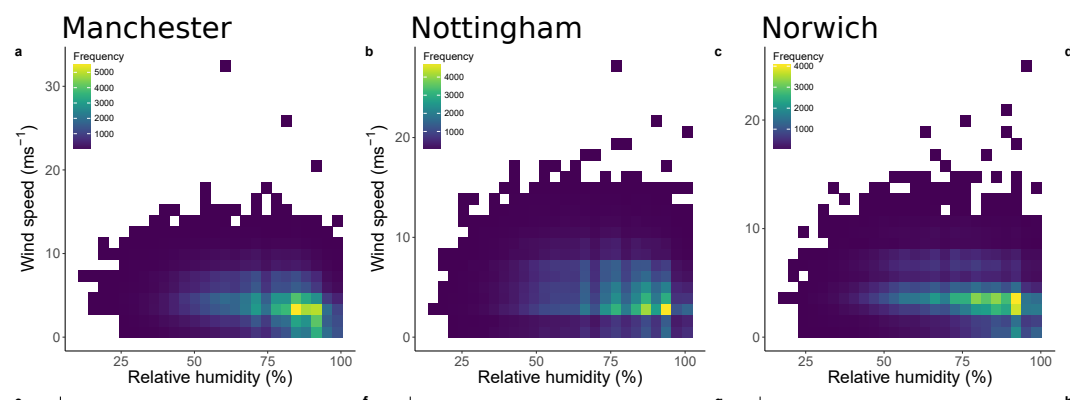

\section{Exeter}
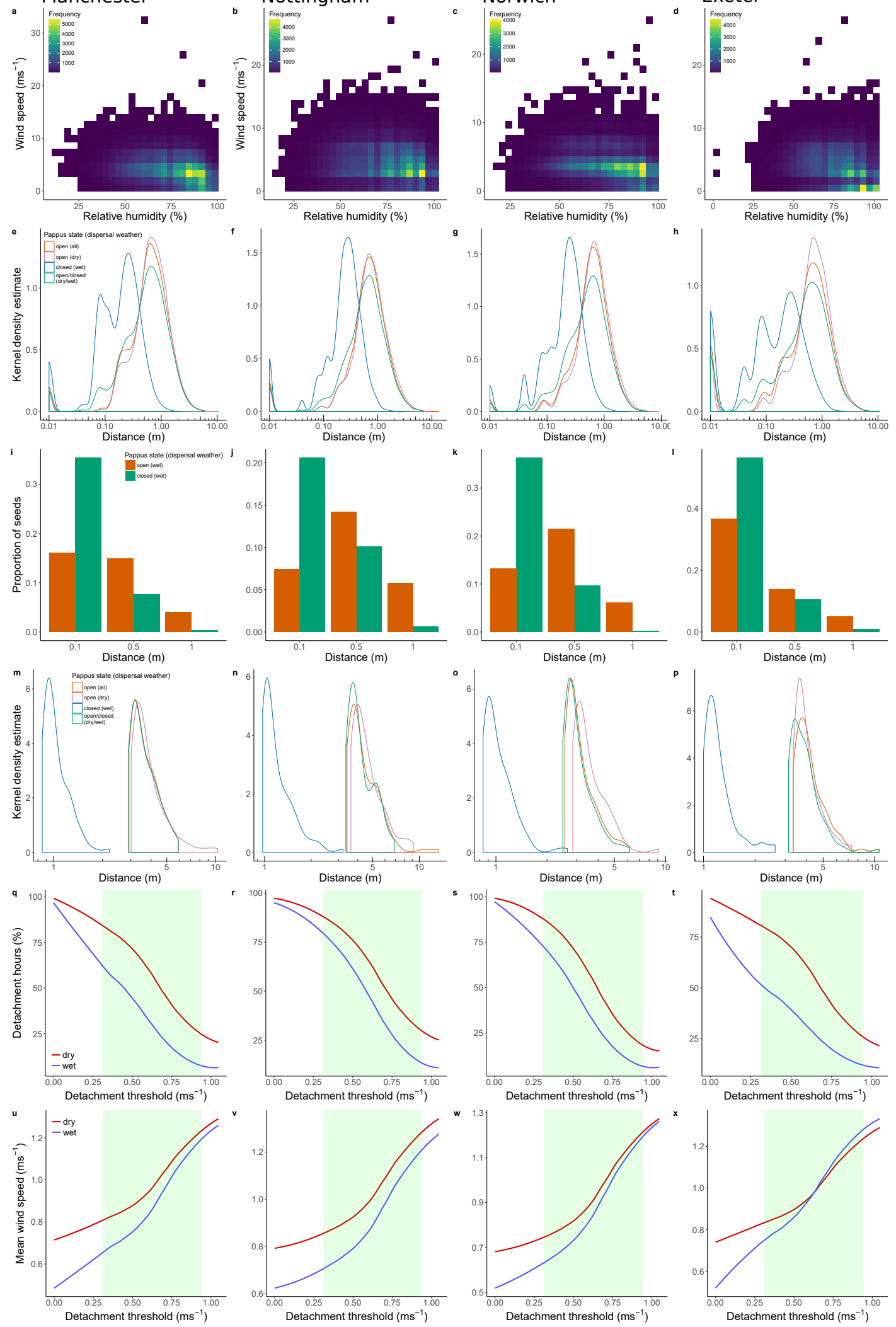

Fig. S3. Supplementary Figure 3. Same plots as Fig 3a-f corresponding to Manchester (a,e,i,m,q,u), Nottingham (b,f,j, ,n,r,v), Norwich $(\mathbf{c}, \mathbf{g}, \mathbf{k}, \mathbf{o}, \mathbf{s}, \mathbf{w})$ and Exeter( $\mathbf{d}, \mathbf{h}, \mathbf{l}, \mathbf{p}, \mathbf{t}, \mathbf{x})$. 
bioRxiv preprint doi: https://doi.org/10.1101/542696; this version posted August 26,2020 . The copyright holder for this preprint (which was not certified by peer review) is the author/funder. All rights reserved. No reuse allowed without permission.
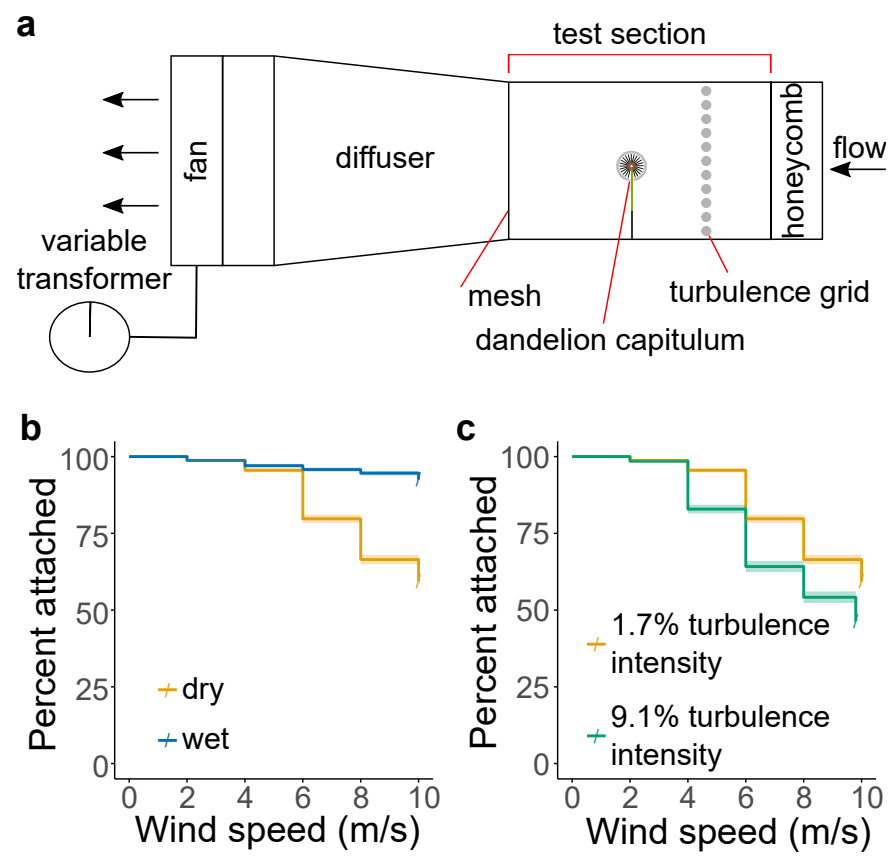

Fig. S4. Supplementary Figure 4. a schematic illustrating experimental setup for detachment assays, bsurvival plot of percentage of fruits attached to capitula at varying wind speeds for $1.7 \%$ turbulent intensity flow in dry and wet conditions, tested 3 days after opening of capitula, $n=21$ capitula per treatment corresponding to 3736 and 3652 seeds for dry and wet conditions respectively, shading around lines indicates s.e.m. c survival plot of percentage of fruits attached to capitula at varying wind speeds for dry conditions with turbulent intensities of $1.7 \%$ and $9.1 \%$, tested 3 days after opening of capitula, (data for $1.7 \%$ turbulent intensity are the same as for dry capitula in panel b), $n=20$ capitula for the $9.1 \%$ turbulent intensity treatment corresponding to 2677 seeds, shading around lines indicates s.e.m. 